\title{
Wie viel Körper braucht der Mensch?
}

\author{
Erhard Taverna
}

Die Körber-Stiftung, gegründet 1959, mit Sitz in Hamburg, fördert die Alltagskultur der Demokratie. Ihr Ziel ist die Erprobung neuer Ideen und Methoden und deren gesellschaftlicher Austausch. Sie möchte andere anstiften, selbst Initiative zu ergreifen. Dazu lockt sie mit Preisen $\mathrm{zu}$ originellen Wettbewerben, für Jugendliche, die vor Ort über Geschichte forschen, für Studierende, für Fotografen, für die europäische Wissenschaft oder für praxisnahe transatlantische Ideen. Die Publikationen des Verlags vertiefen oder ergänzen das reiche Material der prämierten Arbeiten. Zum Beispiel mit der Edition unkonventioneller Reflexionen zum Thema «Wie viel Körper braucht der Mensch?», einer Beitragssammlung zum gleichnamigen Wettbewerbsthema «Bodycheck» von 2001, zugänglich unter www.studienpreis.de.

\section{Der Wettbewerb}

Alle zwei Jahre wird ein neues Thema von aktueller gesellschaftlicher Bedeutung zur Debatte gestellt. Als Beispiel soll hier kurz eine prämierte Arbeit aus der Pflegewissenschaft vorgestellt werden: «Der Körper als Verlust- und Fundsache in Krankenhaus und Pflegeheim - Störungen der Orientierung am eigenen Körper.» Darin geht es um den Verlust des permanenten Prozesses der Selbstaktualisierung, das heisst der kontinuierlichen Versorgung mit Sinnesreizen. Die Autorin entwickelt Ideen und Anleitungen zum Vorbeugen gravierender Orientierungsverluste bei Personen, die am Mangel von Bewegungsund Tastinformationen leiden. Die Thematik des gestörten Köperschemas wird aus neurophysiologischer, pflegerischer und psychologischer Sicht bearbeitet. Anschaulich und präzis beschrieben sind die reizdeprivierenden Situationen im Heim und Spital und die lagerungsbedingten Veränderungen der Wahrnehmung bei Immobilität, bei mangelnder vestibulärer Stimulation oder durch superweiche Lagerung. Der lösungsorientierte Teil der Arbeit ist der körperschemarekonstruierenden Pflege gewidmet, den Mitteln der propiozeptiven Stimulation, der Bewegungsrealisierung, der Musik, der imaginativen Prozesse, der taktilen Stimulation oder der Wiederherstellung eines Raumschemas. Gemeint sind motorisches Üben nach Felden- krais, Bewegungsübungen nach Bobath oder Fröhlich oder Herstellen eines Milieus, das optisch, akustisch und olfaktorisch stimuliert. Mit Melodien und Rhythmen können einmal erlernte Abläufe aktiviert werden oder Komatösen Struktur und Sicherheit vermitteln. Das mentale Bewegen immobiler Körperpartien provoziert EEG-Potentiale, die den realen ähnlich sind, was auch von aussen mit Hilfe einer Puppe (Pfeifenputzerfigur) helfen kann, die Motorik anzubahnen. Wenn es gelingt, die Aufmerksamkeit zu wecken, kann eine «reizende» Umwelt animieren, oder ausserhalb des Patientenblickfeldes sogar eine Bewegung provozieren. Grosse Spiegel sollen, wie in der Sportpädagogik üblich, ein Raumschema rekonstruieren helfen. Schaukelbewegungen und Körperkontakte durch Angehörige nützen Positionsreize. Besonders illustrative Fallbeschreibungen von Oliver Sacks erklären die detaillierten Vorschläge. Der verdiente Preis geht an eine beispielhafte, gelungene Antwort zur gestellten Preisfrage, mit praktischen Konsequenzen für eine ganze Berufsgruppe. Gleich wie die angewandte Robotik, die den Körper als Voraussetzung für logischmathematische Intelligenz entdeckt hat, werden hier die Voraussetzungen zur räumlichen und körperlich-kinästhetischen Intelligenz für den medizinischen Alltag beschrieben.

\section{Das Buch}

17 Autorinnen und Autoren, darunter der Herausgeber Gero von Randow, Buchautor und Ressortleiter bei der Frankfurter Allgemeinen Zeitung, liefern die Theorie zum breiten Spektrum Bodycheck. Als einziger Vertreter der Schweiz äussert sich der Geschichtsprofessor von der Universität Zürich, Prof. Dr. Jakob Tanner, zu Körpermaschinen in der Arbeitsgesellschaft - «Be a somebody with a body». Dem Editorial wurde ein Zitat von Georg Christoph Lichtenberg vorangestellt: «Das Ding, von dessen Augen und Ohren wir nichts und von dessen Nase und Kopfe wir nur sehr wenig sehen, kurz unser Körper.»

Das Interesse an diesem Ding und das Wissen darüber haben gewaltig zugenommen. Medizinern besonders empfohlen sei eine Analyse des Mathematikers und Robotikspezialisten 
Herbert Jaeger. Wer dieses Teamspiel der Neuroforschung in seinem Badezimmer erlebt hat, wird nie mehr mit der bisherigen Selbstverständlichkeit seine Zahnbürste in die Hand nehmen. Gesellschaftliche Körperinszenierungen, der politische Körper, der Körper als Baustelle und die Auswertung einer Umfrage erkunden weitere Perspektiven. Das Ding ist wieder einmal Inspiration, Fundgrube und unerschöpfliche Quelle für immer neue soziale, historische und medizinische Entdeckungsreisen.
- Gunda Rosenberg, Studiengang: Lehramt Pflegewissenschaft, Universität Bremen, 9./10. Semester, der Körper als Verlust- und Fundsache in Krankenhaus und Pflegeheim, 48 Seiten, 2001, E-Mail: rosenb@uni-bremen.de

- Wie viel Körper braucht der Mensch? Standpunkte zur Debatte, für den deutschen Studienpreis herausgegeben von Gero von Randow, edition Körber-Stiftung, Hamburg 2001, 198 Seiten. 